\title{
Targeting cancer stem cells in cancer therapy
}

\author{
Chang Zhou ${ }^{1 \mathrm{a}}$, Hong-Yuan Chen ${ }^{1 \mathrm{~b}}$, Hong-Ce Chen ${ }^{1 \mathrm{c}}$ Wen Rui ${ }^{2^{\star} \mathrm{d}}$ \\ ${ }^{1}$ School of Basic Courses, Guangdong Pharmaceutical University, Guangzhou 510060, P.R. China \\ ${ }^{2}$ Centre Laboratory, Guangdong Pharmaceutical University, Guangzhou 510006, P.R. China \\ *Corresponding author: Centre Laboratory, GuangDong Pharmaceutical University, 280 Waihuan \\ East Road, Guangzhou Higher Education Mega Center, Guangzhou 510006, P.R. China \\ a 289550084@qq.com, ${ }^{\text {b }}$ hcchen@gdpu.edu.cn, ${ }^{\text {c }}$ hcchen2009@126.com, ${ }^{\text {d }}$ gyruiwen@gdpu.edu.cn
}

Keywords: Cancer stem cell, Immunotherapy, Tumor microenvironment, Non-coding RNA, Tumor associated genes.

Abstract. Cancer stem cells (CSCs) have emerged as a population of cells that plays a vital role in recurrence of metastatic cancer, particularly resistant to chemotherapy and radiotherapy. In this article, we will discuss the correlation of CSCs with tumor immunology, microenvironment, long noncoding RNAs (lncRNAs) and tumor associated genes. These particular areas of discussion may help to identify novel strategies to target CSCs in future cancer therapies.

\section{Introduction}

Cancer stem cells (CSCs) are cancer cells which has the characteristics of stem cells. CSCs are very similar to stem cells in many aspects, such as self-renewal, unlimited proliferation and the asymmetric division capacity ${ }^{[1]}$. CSCs are lack of pure mature energy power, they tend to accumulate replication errors ${ }^{[2]}$. On the base of these analysis, CSCs have been defined as a small sub-population of "tumor-initiating cells" or "tumorigenic cells" which may be the key contributors to maintenance, recurrence, and metastasis of cancer ${ }^{[3]}$. CSCs were conducted in ALL ${ }^{[4]}$ AML $^{[5]}$ and other solid cancers. And CSCs model provides an explanation for the resistance to chemotherapy in some type of tumors ${ }^{[6]}$. Expression of CD133 in glioblastoma cell population increased after radiation therapy, whether in glioma cell lines or in nude mice ${ }^{[7]}$.

Therefore, strategies that aim to CSCs are of particular interest in cancer therapy ${ }^{[8]}$. This study conducts a review of published reports to identify the mechanisms of therapies for targeting CSCs.

\subsection{The Immune System and CSCs}

Harnessing the power of the immune system against CSCs, has become an area of significant interest ${ }^{[9]}$. Cellular immune-based therapies targeting CSCs have shown that cytotoxic $\mathrm{T}$ lymphocytes (CTLs) are specific for the tumor-associated antigen CEP55 can efficiently recognize CSCs. Moreover, intentional activation of Vgamma9Vdelta2 T cells by zoledronate may increase anti-tumor activities by inducing the production of cytokines (TNF-alpha and IFN-gamma) to destroy $\operatorname{CSCs}^{[10]}$.

Vaccines provide a unique avenue to utilize the immune system in generating anti-tumor activities. A novel melanoma B16F10-ESAT6-gpi/IL-21 CD133(+)CD44(+) CSC vaccine has been shown to exhibit anti-melanoma lung metastasis efficacy and was associated with elevated levels of serum anti-antigenic target (ESAT6) and IFN-gamma and increased cytotoxic activities of natural killer cells ${ }^{[11]}$.

Antibody constructs provide another approach to act against CSCs. The anti-EREG antibody (epiregulin, epidermal growth factor family) has been shown to exhibit significant efficacy in an established metastatic NOG (NOD/Shi-scid/IL-2Rcnull) mouse model. The anti-EREG antibody is particularly effective in the early stage of tumorigenesis when the cancer is rich in $\mathrm{CSCs}^{[12]}$.

\subsection{The Microenvironment and CSCs}

CSCs maintain characteristics though interacting with their microenvironment. One of the components of CSCs niche is the tumor-associated macrophage (TAM) ${ }^{[13]}$. In rat C6 glioma side population (SP) cells as a model of glioma CSCs, CSC-induced macrophages can be separated into 
CD11 $c^{\text {low }}$ and CD11c ${ }^{\text {high }}$ cells. Intracranial transplantation into immune-deficient mice together with CSCs showed that only the CD11 $c^{\text {high }}$ subset of cells possessed protumoral activity. Therefore, these CD11c ${ }^{\text {high }}$ macrophages may be a target in disrupting the CSC niche.

Cytokines (TGF- $\beta$, TNF- $\alpha$, IL-6) produced by the microenvironment can provide signals to induce self-renewal of CSCs ${ }^{[14]}$. IL-6 plays important roles in CSC maintenance. Currently, non-small cell lung cancer (NSCLC) CD133+ cells expressing IL-6 exhibited increased survival following radiation treatment comparing to those with knockdown of IL-6 expression. IL-6 plays an important role in protecting NSCLC CD133+ cells from radiation-induced DNA damage and apoptosis $^{[15]}$.These findings suggest that modulating IL-6 activity may provide an effective therapeutic strategy in NSCLC CSCs treatment.

\subsection{Non-coding RNA and CSCs}

Non-coding RNA, including microRNA (miRNA) and long non-coding RNA, can alter the properties of CSCs and may provide novel therapeutic targets. One study utilized PCR-based miRNA profiling analysis of normal colon stem cell (NCSCs) and colon CSCs (EpCAM+/CD44+/CD66a-) to identify miR-137 (miRNA-137), which serves to regulate colon CSC properties ${ }^{[16]}$. MiR-137 was downregulated in the colon CSCs compared to NCSCs. Overexpression of miR-137 suppressed the development of colon cancer in vitro and the tumorigenicity of colon cancer cells in vivo through inhibition of DCLK1 expression.

Recently, long non-coding RNAs (lncRNAs) have been reported to exhibit aberrant expression in various malignancies of CSCs. Depletion of lnc-DILC has been shown to markedly enhance expansion of liver cancer stem cells (LCSCs) and facilitate hepatocellular carcinoma (HCC) initiation and progression. Furthermore, lnc-DILC overexpression suppressed the effects of IL-6 transcription, signal transducer and activator of transcription 3 (STAT3) activation, and LCSC expansion triggered by lnc-DILC depletion ${ }^{[17]}$. An oncogenic lncRNA, MALAT-1, has been reported to promote EMT in pancreatic cancer and to regulate expression of CSC markers ${ }^{[18]}$. MALAT1 could increase the expression pancreatic CSCs, maintain self-renewing capacity, decrease sensitivity to anticancer medications, and accelerate tumor angiogenesis in vitro and in vivo. The potential effects of MALAT1 suggest it will be as a promising therapeutic target for pancreatic CSCs.

\subsection{Stemness associated genes and CSCs}

Sox2, Bmi1, and Oct4 have been identified as stemness associated genes of CSCs. These genes and their specific signaling pathways have been suggested to contribute to the stem cell features of $\mathrm{CSCs}^{[19]}$. Modulation of stemness associated genes may provide a potential molecular target for suppressing the proliferative and resistant properties of CSCs. Bmi1 (B-cell-specific Moloney murine leukemia virus insertion site 1) is a member of the Polycomb group gene (PcG) family, which plays a role in the proliferation, migration, and tumorigenesis of several types of CSCs. Knockdown of Bmi1 by shRNA in CD44+ nasopharyngeal carcinoma cancer stem-like cells inhibited tumor proliferation, migration, and invasion in vitro and in vivo. Furthermore, silencing Bmi-1 expression with shRNA increased tumor apoptosis through the p16INK4a-p14ARF-p53 pathway $^{[20]}$.

\section{Conclusion}

The CSC theory has provided an insight into the functions of CSCs. CSCs can make these cells appeale targets in drug design and cancer treatment including their immunology, interactions with their microenvironments, their relationships between non-coding RNA, and the role of stemness associated genes. As the complexity of CSCs, multiple therapeutic strategies may need to be combined to synergistically optimize and improve CSC targeting and future cancer therapy.

\section{References}

[1]. P Valent, D Bonnet, RD Maria, T, et al. Cancer stem cell definitions and terminology: the devil is in the details. Nat. Rev. Cancer.Vol.12(2012),p.767-775. 
[2]. PB Gupta, CL Chaffer, RA Weinberg. Cancer stem cells: mirage or reality? Nature Med. Vol.15(2009),p.1010-1012.

[3]. Puglisi MA, Tesori V, Lattanzi W, Gasbarrini GB, Gasbarrini A. Colon cancer stem cells: controversies and perspectives. World J Gastroenterol. Vol.19(2013),No.20,p.2997-3006.

[4]. Kamelreid S, Letarte M, Sirard C,et al. A Model of Human Acute Lymphoblastic-Leukemia in Immune-Deficient Scid Mice. Science. Vol.246(1989) ,p.1597-1600.

[5]. Lapidot T, Sirard C, Vormoor J, et al. A Cell Initiating Human Acute Myeloid-Leukemia after Transplantation into Scid Mice.Nature. Vol.367(1994), p.645-648.

[6]. Eramo A, Lotti F, Sette G, Pilozzi E,et al. Identification and expansion of the tumorigenic lung cancer stem cell population.Cell Death Differ. Vol.15(2008),p.504-514.

[7]. Tamura K, Aoyagi M, Ando N, et al. Expansion of CD133-positive glioma cells in recurrent de novo glioblastomas after radiotherapy and chemotherapy. J Neurosurg. Vol.119(2013),No.5,p.1145-1155.

[8]. Noguchi K,Konno M,Nishida N, et al. Therapeutic Implication Targeting for Cancer Stem Cells. Gan To Kagaku Ryoho. Vol.42(2015),No.9,p.1036-1039.

[9]. Schreiber RD, Old LJ, Smyth MJ. Cancer immunoediting: integrating immunity's roles in cancer suppression and promotion. Science. Vol.331(2011),p.1565-1570.

[10]. Todaro MD, Asaro M, Caccamo N, et al. Efficient killing of human colon cancer stem cells by gammadelta T lymphocytes. J Immunol.Vol.182(2009),No.11,p.7287-7296.

[11]. Zhao F, He X, Sun J , et al. Am J Transl Res.Cancer stem cell vaccine expressing ESAT-6-gpi and IL-21 inhibits melanoma growth and metastases.Vol.7(2015),No.10,p.1870-1882.

[12]. Zhong H, Davis A, Ouzounova M, et al. A Novel IL6 Antibody Sensitizes Multiple Tumor Types to Chemotherapy Including Trastuzumab-Resistant Tumors. Cancer Res. Vol.76(2016),No.2,p.480-490.

[13]. Kokubu Y, Tabu K, Muramatsu N, et al. Induction of protumoral CD11chigh macrophages by glioma cancer stem cells through GM-CSF. Genes to Cells.Vol.21(2016),No.3,p.241-251.

[14]. Sugong Chen, Emina H Huang. The Colon Cancer Stem Cell Microenvironment Holds Keys to Future Cancer Therapy. J Gastrointest Surg. Vol.18( 2014),No.5,p. 1040-1048.

[15]. Chen Y, Zhang F, Tsai Y, et al. IL-6 signaling promotes DNA repair and prevents apoptosis in CD133+ stem-like cells of lung cancer after radiation.Radiat Oncol. Vol.10(2015),p.227.

[16]. Sakaguchi M, Hisamori S, Oshima N, et al. miR-137 Regulates the Tumorigenicity of Colon Cancer Stem Cells Through the Inhibition of DCLK1. Mol Cancer Res.Vol.14(2016),No.4,p.354-362.

[17]. Wang X, Sun W, Shen W, et al. Long Non-coding RNA DILC Represses Self-renewal of Liver Cancer Stem Cells via Inhibiting Autocrine IL-6/STAT3 Axis.Journal of Hepatology Vol.64(2016).No.6,p.1293-1294

[18]. Jiao F, Hu H, Han $\mathrm{T}$, et al. Long noncoding RNA MALAT-1 enhances stem cell-like phenotypes in pancreatic cancer cells.Int J Mol Sci. Vol.16(2015),No.4,p.6677-6693.

[19]. Xu MH, Gao X, Luo D, et al. EMT and acquisition of stem cell-like properties are involved in spontaneous formation of tumorigenic hybrids between lung cancer and bone marrow-derived mesenchymal stem cells. PLoS One.Vol.9(2014),No.2,p.87893.

[20]. Xu X, Liu Y, Su J, et al.Downregulation of Bmi-1 is associated with suppressed tumorigenesis and induced apoptosis in CD44+ nasopharyngeal carcinoma cancer stem-like cells. Oncol Rep. Vol.35(2016).No.2,p.923-931. 